stimulation and by hyperventilation in 7 (58\%). JS patients show focal posterior interictal EDs and focal posterior ictal EDs preceding generalized EDs. The findings suggest that seizures and EEG paroxysms induced by eye closure, intermittent photic stimulation, and hyperventilation are indicative of the occipital cortex initiating the generalized epilepsy network in JS. (Viravan S, Go C, Ochi A, Akiyama T, Snead OC III, Otsubo H. Jeavons syndrome existing as occipital cortex initiating generalized epilepsy. Epilepsia July 2011;52(7):1273-1279). (Respond: Hiroshi Otsubo MD, Division of Neurology, The Hospital for Sick Children, 555 University Avenue, Toronto, ON M5G 1X8, Canada. Email: hiroshi.otsubo@sickkids.ca).

COMMENT. Jeavons syndrome (JS) is referred to as an idiopathic "eye closureinduced" reflex idiopathic generalized epilepsy. In the 2006 ILAF classification of epileptic syndromes, JS was not included as an epileptic syndrome, but eyelid myoclonia was classified as a generalized seizure. Panayiotopoulos (2005) proposed the inclusion of JS as a reflex epilepsy, and Striano et al (2009) as an idiopathic generalized epilepsy. Under the revised terminology (2005-2009) for the ILAE, proposed by Berg AT et al (Epilepsia 2010;51(4):676-685), JS would probably be included as an electroclinical syndrome and a distinctive "constellation" of "genetic" or "unknown" etiology. Four (33\%) of the patients in the above series had a family history of epilepsy.

\title{
PREDICTORS OF SEIZURE FREEDOM AFTER SURGERY FOR CORTICAL MALFORMATIONS
}

Researchers at the Northern California Epilepsy Center and University of California, San Francisco, conducted a retrospective review of 143 patients with malformations of cortical development (MCD) who underwent resection surgery for medically refractory epilepsy. Median age of seizure onset was 7 years (range, 0-55 years) with $123(86 \%)$ having childhood onset $(<18$ years $)$. Complex partial seizures were most common (47.5\%) followed by generalized tonic-clonic (23\%). Preoperative MRI, classified according to a new MCD classification, included subcortical T2/FLAIR abnormality $(46.1 \%)$, blurring of gray-white junction (26.6\%), cortical thickening $(17.2 \%)$, and mesiotemporal sclerosis (16.1\%). ECoG revealed epileptiform activity in 111 patients, focal in $67.7 \%$. Total resection of abnormal area was achieved in $68.1 \%$. Heterotopia were present in $35 \%$, and neuronal dysplasias in $33.3 \%$. According to the Barkovich classification for MCD, 22\% were class I (abnormal neuronal and glial proliferation), 10\% class II (abnormal migration), 65\% class III (abnormal organization), and 3\% class IV (abnormal development). Class I patients had better outcome than class II or III. Complete resection of ECoG and anatomic abnormalities was the main independent predictor of seizure freedom. Other favorable predictors were smaller lesions and locally confined ECoG abnormalities. Long-term follow-up demonstrated seizure control in $72 \%$ at 2 years, $65 \%$ at 5 years, and $67 \%$ at 10 years. (Chang EF, Wang DD, Barkovich AJ et al. Predictors of seizure freedom after surgery for malformations of cortical development. Ann Neurol July 2011;70(1):151-162). (Respond: Dr Chang, Department of Neurological Surgery, University of California, San Francisco, 505

Parnassus Avenue, M779, San Francisco, CA 94143. E-mail: changed@neurosurg.ucaf.edu). 
COMMENT. MR imaging and intracranial ECoG recordings are essential determinants of outcome of patients with MCD and medically intractable epilepsy. Neuronal proliferation pathology carries a better prognosis than abnormalities of cortical migration or organization. Surgery can result in $67 \%$ long-term seizure freedom.

\section{MEMORY DEFICITS IN CHILDREN WITH MESIAL TEMPORAL SCLEROSIS AND EPILEPSY}

Researchers at Sao Paulo School of Medicine, Brazil, analyzed the occurrence of episodic memory (for personally experienced events) and semantic memory (for stored knowledge acquired in the past) deficits in 19 consecutive children with mesial temporal sclerosis (MTS) (8-16 years old; mean IQ 97). Patients performed worse on tests of immediate and delayed verbal episodic memory, visual episodic memory, verbal and visual learning, mental scanning for semantic clues, object naming, word definition, and repetition of sentences. Patients with a history of status epilepticus had worse visual episodic memory, whereas patients with uncontrolled daily and weekly seizures had worse verbal learning. Patients on polytherapy were more impaired in visual learning. Early age of seizure onset had a significant negative impact on semantic memory tests. Except for a lower score on the Boston Naming Test with left sided MTS cf right, episodic or semantic memory tests showed no differences with laterality of MTS. (Rzezak P, Guimaraes C, Fuentes D, Guerreiro MM, Valente KDR. Episodic and semantic memory in children with mesial temporal sclerosis. Epilepsy Behav July 2011;21:242-247). (Respond: Dr Patricia Rzezak, Rua Abdo Ambuba, 75/31 Sao PauloSP, Brazil, 05725-030. E-mail: patriciarzezak@gmail.com).

COMMENT. MTS epilepsy in children is associated with significant deficits in episodic and semantic memory function, despite normal intelligence. The earlier the onset of epilepsy, the more severe is the impairment of semantic memory. Definition of distinct domains of memory is required for rehabilitation measures.

Tests of everyday verbal memory in $\mathbf{1 3 2}$ children with epilepsy were predictive of academic performance but not significantly correlated with reports of prospective memory. (Chapieski L et al. Epilepsy Behav July 2011;21:285-290).

\section{ATTENTION DEFICIT DISORDERS}

\section{EFFICACY AND SAFETY OF METHYLPHENIDATE FOR ADHD AND UNCONTROLLED SEIZURES}

Twenty-four participants with uncontrolled epilepsy and ADHD in this prospective study, conducted at University of Joinville, Santa Catarina, Brazil, were 7 16 years of age. They had been followed for at least 6 months before introduction of methylphenidate (MPH), and had at least 2 seizures treated with antiepileptic drugs. MPH dose started at $5 \mathrm{mg}$ once or twice daily, and was increased by $10 \mathrm{mg}$ weekly as necessary, not exceeding $60 \mathrm{mg}$ daily. MPH was effective in control of ADHD in 70.8\% 